\title{
Existence of Matrices With Prescribed Off-Diagonal Block Element Maxima
}

\author{
J. A. Dias Da Silva*†
}

Departamento de Matemática

da Universidade de Lisboa

Rua Ernesto de Vasconcelos, Bloco C1

1700 Lisboa, Portugal

Daniel Hershkowitz $\ddagger$

\section{Mathematics Department}

Technion-Israel Institute of Technology

Haifa 32000, Israel

and

Hans Schneider ${ }^{\ddagger}$

Mathematics Department

University of Wisconsin-Madison

Madison, Wisconsin 53706

Submitted by Helene Shapiro

\section{ABSTRACT}

Necessary and sufficient conditions are proven for the existence of a real square matrix such that for every principal submatrix the maximal (or minimal) value of an element in the row complement of the submatrix is prescribed. The problem is solved in the cases where the positions of the nonzero elements of $A$ are contained

*The research of all three authors was supported by the Fundação Calouste Gulbenkian, Lisboa.

tThe research of this author was carried out within the activity of the Centro de Álgebra da Universidade de Lisboa.

¥ The research of these authors was supported by their joint grant 90-00434 from the United States-Israel Binational Science Foundation, Jerusalem, Israel.

$\S$ The research of this author was supported in part by NSF grants DMS-9123318, DMS-8901445, and EMS-8718971. 
in a given set of positions, where the positions of the nonzero elements of $A$ are all given, and where there is no restriction on the positions of the nonzero elements of $A$. The uniqueness of the solution is studied as well.

\section{INTRODUCTION}

In analogy with well-studied problems concerning the existence of nonnegative matrices with a prescribed pattern and with prescribed row and columns sums (e.g. [7, 1, 3, 4] and many other papers), Rothblum, Schneider, and Schneider [5] have recently studied the existence of matrices with a prescribed pattern and prescribed row and column maxima. Further, it is clear that the conditions for existence determined in [5] force certain relations on the maxima of elements in the off-diagonal submatrices. In this paper, we find necessary and sufficient conditions for the existence of a real matrix with prescribed pattern and prescribed maxima of the elements in all off-diagonal blocks.

In most of the papers referenced above the existence problems studied were motivated by scaling problems. Another such scaling problem, namely the max-balancing problem, was considered in [6]. There it is pointed out that, in contrast with the corresponding problem for sums, max-balancing the rows and columns of a matrix does not imply max-balancing symmetrically. placed off-diagonal blocks. Thus the authors were led to prove a condition for max-balancing off-diagonal blocks. This is an additional motivation for our study.

We now describe the paper in some more detail.

In Section 2 we solve three levels of the existence problem:

(i) The case in which the positions of the nonzero elements of $A$ are contained in a given set of positions.

(ii) The case in which the positions of the nonzero elements of $A$ are all given.

(iii) The case in which we have no restriction on the positions of the nonzero elements of $A$ (the "graph-free" case).

Both the maxima and the minima versions of all three cases are fully characterized.

The existence problems do not always have a unique solution. In Sec- 
tion 3 we study the uniqueness of the solutions for the problems discussed in Section 2. We define the ROWB algorithm for removal of certain arcs in a digraph, and then show that a given pattern yields a unique solution for every set of given maxima if and only if the $R O W B$ algorithm terminates after one iteration at most. A given pattern yields a unique solution for some set of given maxima if and only if the ROWB algorithm terminates after a finite number of iterations.

Motivated by the results of Section 4 , Section 5 is devoted to a short discussion of digraphs for which the ROWB algorithm terminates after one iteration at most.

This paper is a companion paper to [2], where we solve the analogous problem with sums in place of maxima.

\section{THE EXISTENCE PROBLEM}

In this section we discuss the existence of a matrix such that for every principal submatrix we prescribe the maximal value of an element in the row complement of the submatrix.

We start with a few definitions.

Notation 2.1. We denote by $\langle n\rangle$ the set $\{1, \ldots, n\}$.

\section{DEFINITION 2.2 .}

(i) Let $D=(V, E)$ be a digraph, and let $S$ and $T$ be disjoint subsets of $V$. We denote by $\widetilde{S}_{T}$ the set $\{i \in S:(i, j) \in E$ for some $j \in T\}$.

(ii) We denote by $\widetilde{S}$ the set $\widetilde{S}_{S^{c}}$, where $S^{c}$ denotes the complement of $S$ in $V$. We say that $S$ is a relevant subset of $V$ if $\widetilde{S}$ is nonempty.

(iii) We denote by $S^{\perp}$ the maximal subset $T$ of $S^{c}$ such that $\widetilde{S}_{T}=\emptyset$.

Definition 2.3. Let $A$ be an $n \times n$ matrix. The digraph $D(A)$ of $A$ is defined as the digraph with vertex set $\langle n\rangle$, and where $(i, j)$ is an arc in $D(A)$ if and only if $a_{i j} \neq 0$.

Definition 2.4. Let $D=(V, E)$ be a digraph. A digraph $D^{\prime}=$ $\left(V^{\prime}, E^{\prime}\right)$ is said to be a subdigraph of $D$ if $V^{\prime} \subseteq V$ and $E^{\prime} \subseteq E$. We write $D^{\prime} \subseteq D$ to indicate that $D^{\prime}$ is a subdigraph of $D$.

CONVENTION 2.5. The maximum of the elements of the empty subset of the reals will be considered to be $-\infty$. 
The main result in this section is

THEOREM 2.6. Let $\left\{X_{S}: S \subseteq\langle n\rangle\right\}$ be a set of real numbers, and let $D=(\langle n\rangle, E)$ be a digraph. The following are equivalent:

(i) There exists an $n \times n$ matrix $A$, with $D(A) \subseteq D$, such that

$$
\max _{(i, j) \in S \times S^{c} \cap E}\left\{a_{i, j}\right\}=X_{S}, \quad S \subseteq\langle n\rangle .
$$

(ii) We have

$$
\begin{aligned}
X_{\emptyset} & =X_{\langle n\rangle}=-\infty, & & \\
X_{S} & \leq \max \left\{X_{S \cup T}, X_{S^{\prime}}\right\}, & & S, T \subseteq\langle n\rangle, \quad S \cap T=\emptyset, \\
X_{S \cup T} & \leq \max \left\{X_{S}, X_{T}\right\}, & & \widetilde{S}_{T} \subseteq S^{\prime} \subseteq S \\
X_{S \cap T} & \leq \max \left\{X_{S}, X_{T}\right\}, & & S, T \subseteq\langle n\rangle,
\end{aligned}
$$

Proof. (i) $\Rightarrow$ (ii): In view of Convention $2.5,(2.8)$ follows immediately from (i). Let $S$ and $T$ be disjoint subsets of $\langle n\rangle$. By Definition 2.2, it follows that

$$
\begin{aligned}
\left(S \times S^{c}\right) \cap E & =[(S \times T) \cap E] \cup\left(\left[S \times(S \cup T)^{c}\right] \cap E\right) \\
& =\left[\left(S^{\prime} \times T\right) \cap E\right] \cup\left(\left[S \times(S \cup T)^{c}\right] \cap E\right) \\
& \subseteq\left[\left(S^{\prime} \times S^{\prime c}\right) \cap E\right] \cup\left(\left[(S \cup T) \times(S \cup T)^{c}\right] \cap E\right) .
\end{aligned}
$$

Therefore, clearly (i) implies (2.9). Now let $S$ and $T$ be subsets of $\langle n\rangle$. It follows from (i) that

$$
\begin{aligned}
X_{S \cup T} & =\max _{(i, j) \in\left[S \cup T \times\langle S \cup T)^{c}\right] \cap E}\left\{a_{i j}\right\} \\
& =\max \left\{\max _{(i, j) \in\left[S \times(S \cup T)^{c}\right] \cap E}\left\{a_{i j}\right\}, \max _{(i, j) \in\left[T \times(S \cup T)^{c}\right] \cap E}\left\{a_{i j}\right\}\right\} \\
& \leq \max \left\{\max _{(i, j) \in S \times S^{c} \cap E}\left\{a_{i j}\right\}, \max _{(i, j) \in T \times T^{c} \cap E}\left\{a_{i j}\right\}\right\} \\
& =\max \left\{X_{S}, X_{T}\right\} .
\end{aligned}
$$

Similarly, we have

$$
X_{S \cap T}=\max _{\langle i, j) \in[S \cap T \times(S \cap T) c] \cap E}\left\{a_{i j}\right\}=\max _{(i, j) \in\left[S \cap T \times\left(S^{c} \cup T^{c}\right)\right] \cap E}\left\{a_{i j}\right\}
$$




$$
\begin{aligned}
& =\max \left\{\max _{(i, j) \in\left[S \cap T \times S^{c}\right) \cap E}\left\{a_{i j}\right\}, \max _{(i, j) \in\left[S \cap T \times T^{c}\right) \cap E}\left\{a_{i j}\right\}\right\} \\
& \leq \max \left\{\max _{(i, j) \in S \times S^{c} \cap E}\left\{a_{i j}\right\}, \max _{(i, j) \in T \times T^{c} \cap E}\left\{a_{i j}\right\}\right\} \\
& =\max \left\{X_{S}, X_{T}\right\} .
\end{aligned}
$$

(ii) $\Rightarrow$ (i): We construct an $n \times n$ matrix $A$ by

$$
a_{i j}=\left\{\begin{array}{cc}
\min _{\substack{T \subseteq\langle n) \\
(i, j) \in T \times T^{c}}}\left\{X_{T}\right\}, & (i, j) \in E, \\
0, & (i, j) \notin E .
\end{array}\right.
$$

We shall prove that $A$ satisfies (2.7). For $(i, j) \in E$ we denote by $T_{i j}$ a subset of $\langle n\rangle$ such that $(i, j) \in T_{i j} \times T_{i j}^{c}$ and $a_{i j}=X_{T_{i j}}$. Let $S$ be a subset of $\langle n\rangle$. If $S=\emptyset$ or $S=\langle n\rangle$, then (2.7) follows from (2.8). So assume that $S \neq \emptyset,\langle n\rangle$. We have

$$
\begin{aligned}
\max _{(i, j) \in S \times S^{c} \cap E}\left\{a_{i j}\right\} & =\max _{(i, j) \in S \times S^{c} \cap E}\left\{\min _{\substack{T \subseteq\langle n\rangle \\
(i, j) \in T \times T^{c}}}\left\{X_{T}\right\}\right\} \\
& \leq \max _{(i, j) \in S \times S^{c} \cap E}\left\{X_{S}\right\}=X_{S}
\end{aligned}
$$

Let

$$
W_{i}=\bigcap_{\substack{j \in S^{c} \\(i, j) \in E}} T_{i j}, \quad i \in \widetilde{S}
$$

For every element $i \in\langle n\rangle$ we denote by $\operatorname{supp}(i)$ the set $\left\{k \in \widetilde{S}: i \in W_{k}\right\}$. Note that $\operatorname{supp}(i)$ may be empty. We define the equivalence relation in $\langle n\rangle$ by $i \sim j \Leftrightarrow \operatorname{supp}(i)=\operatorname{supp}(j)$. We denote by $C_{i}$ the equivalence class of $i$, and we denote $\operatorname{supp}\left(C_{i}\right)=\operatorname{supp}(i)$. Observe that $i \in W_{i}$ whenever $i \in \widetilde{S}$, and hence

$$
C_{i} \cap \widetilde{S} \subseteq \operatorname{supp}\left(C_{i}\right) \subseteq \bigcap_{j \in \operatorname{supp}\left(C_{i}\right)} W_{j}
$$

We define the sets

$$
T_{i}=\left(\bigcap_{j \in \operatorname{supp}\left(C_{i}\right)} W_{j}\right) \bigcap S, \quad i \in \widetilde{S},
$$


and we now claim that

$$
X_{T_{i}} \leq \max _{(j, k) \in\left(S \times S^{c}\right) \cap E}\left\{\min _{\substack{T \subseteq \leq n) \\(j, k) \in T \times T^{c}}}\left\{X_{T}\right\}\right\}, \quad i \in \widetilde{S}
$$

We prove this claim by a decreasing induction on the cardinality $\left|\operatorname{supp}\left(C_{i}\right)\right|$ of $C_{i}$. Assume first that $\operatorname{supp}\left(C_{i}\right)$ is of maximal cardinality $m$. By (2.14) we have

$$
C_{i} \cap \widetilde{S} \subseteq\left(\bigcap_{j \in \operatorname{supp}\left(C_{i}\right)} W_{j}\right) \bigcap \widetilde{S}
$$

Assume that there exists

$$
v \in\left[\left(\bigcap_{j \in \operatorname{supp}\left(C_{i}\right)} W_{j}\right) \cap \widetilde{S}\right] \backslash C_{i}
$$

Since $v \in \bigcap_{j \in \operatorname{supp}\left(C_{i}\right)} W_{j}$, we have $\operatorname{supp}\left(C_{i}\right) \subseteq \operatorname{supp}(v)$. Furthermore, it follows from $(2.17)$ that $\operatorname{supp}\left(C_{i}\right) \neq \operatorname{supp}(v)$, and hence $|\operatorname{supp}(v)|>$ $\left|\operatorname{supp}\left(C_{i}\right)\right|$, in contradiction to the maximality of $\left|\operatorname{supp}\left(C_{i}\right)\right|$. Therefore, our assumption that there exists $v$ satisfying (2.17) is false, and it follows from (2.16) that

$$
C_{i} \cap \widetilde{S}=\left(\bigcap_{j \in \operatorname{supp}\left(C_{i}\right)} W_{j}\right) \bigcap \widetilde{S}
$$

Now, let $u \in T_{i}$ and let $v \in S^{c}$. If $(u, v) \in E$ then $u \in \widetilde{S}$. By (2.18) we have $u \in C_{i}$. By the construction of our sets $T_{i j}$ we have $u \in T_{u v}$ and $v \notin T_{u v}$, and so $u \in W_{u}$ while $v \notin W_{u}$. Since $u \in C_{i}$ we have $\operatorname{supp}(u)=\operatorname{supp}\left(C_{i}\right)$, and hence, since $v \notin W_{u}$, it follows that $v \notin \bigcap_{j \in \operatorname{supp}\left(C_{i}\right)} W_{j}$. Thus, we have

$$
\left(\left[\left(\bigcap_{j \in \operatorname{supp}\left(C_{i}\right)} W_{j}\right) \cap S\right] \times\left[\left(\bigcap_{j \in \operatorname{supp}\left(C_{i}\right)} W_{j}\right) \cap S^{c}\right]\right) \bigcap E=\emptyset .
$$

In view of $(2.19)$, substitution of the sets $\left(\bigcap_{j \in \operatorname{supp}\left(C_{i}\right)} W_{j}\right) \cap S$, $\left(\bigcap_{j \in \operatorname{supp}\left(C_{i}\right)} W_{j}\right) \cap S^{c}$, and $\emptyset$ for the sets $S, T$, and $S^{\prime}$ in (2.9) respectively 
yields $X_{T_{i}} \leq X_{\bigcap_{j \in \operatorname{supp}\left(C_{i}\right)} W_{j}}$, and by $(2.11)$ we have

$$
\begin{aligned}
X_{T_{i}} & \leq \max _{j \in \operatorname{supp}\left(C_{i}\right)}\left\{X_{W_{j}}\right\} \leq \max _{\substack{j \in \operatorname{supp}\left(C_{i}\right) \\
k \in S^{c} \\
(j, k) \in E}}\left\{X_{T_{j k}}\right\} \\
& =\max _{\substack{j \in \operatorname{supp}\left(C_{i}\right) \\
k \in S^{c} \\
(j, k) \in E}}\left\{\min _{\substack{T \subseteq(n) \\
(j, k) \in T \times T^{c}}}\left\{X_{T}\right\}\right\} \\
& \leq \max _{(j, k) \in\left(S \times S^{c}\right) \cap E}\left\{\min _{\substack{T \subseteq(n) \\
(j, k) \in T \times T^{c}}}\left\{X_{T}\right\}\right\},
\end{aligned}
$$

proving (2.15) in the case that $\operatorname{supp}\left(C_{i}\right)$ is of maximal cardinality $m$. Assume now that (2.15) holds whenever $\left|\operatorname{supp}\left(C_{i}\right)\right|>r$, where $r<m$, and let $i$ be such that $\left|\operatorname{supp}\left(C_{i}\right)\right|=r$. Let $u \in C_{i} \cap S$ and let $v \in S^{c}$. If $(u, v) \in E$ then $u \in \widetilde{S}$. By the construction of our sets $T_{i j}$ we have $u \in T_{u v}$ and $v \notin T_{u v}$, and so $u \in W_{u}$ while $v \notin W_{u}$. Since $u \in C_{i}$, we have $\operatorname{supp}(u)=\operatorname{supp}\left(C_{i}\right)$, and hence, since $v \notin W_{u}$, it follows that $v \notin \bigcap_{j \in \operatorname{supp}\left(C_{i}\right)} W_{j}$. Thus, we have

$$
\left(\left(C_{i} \cap S\right) \times\left[\left(\bigcap_{j \in \operatorname{supp}\left(C_{i}\right)} W_{j}\right) \cap S^{c}\right]\right) \bigcap E=\emptyset .
$$

In view of $(2.20)$, substitution of the sets $T_{i},\left(\bigcap_{j \in \operatorname{supp}\left(C_{i}\right)} W_{j}\right) \cap S^{c}$, and $T_{i} \backslash C_{i}$ for the sets $S, T$, and $S^{\prime}$ in (2.9) respectively yields

$$
X_{T_{i}} \leq \max \left\{X_{\bigcap_{j \in \mathrm{supp}\left(C_{i}\right)} W_{j}}, X_{T_{i} \backslash C_{i}}\right\}
$$

and by (2.11) and the definition of $W_{j}$ we have

$$
X_{T_{i}} \leq \max \left\{\max _{(j, k) \in\left[\operatorname{supp}\left(C_{i}\right) \times S^{c}\right] \cap E}\left\{\min _{\substack{T \subseteq(n) \\(j, k) \in T \times T^{c}}}\left\{X_{T}\right\}\right\}, X_{T_{i} \backslash C_{i}}\right\} .
$$

Let $u \in T_{i} \backslash C_{i}$, First, observe that by the definition of $T_{i}$ we have $\operatorname{supp}(i) \subseteq \operatorname{supp}(u)$. Furthermore, since $u \notin C_{i}$, we have $\operatorname{supp}(i) \neq \operatorname{supp}(u)$, and hence $\left|\operatorname{supp}\left(C_{u}\right)\right|>\left|\operatorname{supp}\left(C_{i}\right)\right|$. Now, let $v \in T_{u}$. Since $\operatorname{supp}\left(C_{i}\right) \subseteq$ 
$\operatorname{supp}\left(C_{u}\right)$, it follows by the definition of $T_{i}$ that $v \in T_{i}$. Also, $\operatorname{supp}\left(C_{i}\right) \subseteq$ $\operatorname{supp}\left(C_{u}\right) \subseteq \operatorname{supp}(v)$, and $\operatorname{since} \operatorname{supp}\left(C_{i}\right) \neq \operatorname{supp}\left(C_{u}\right)$, it follows that $v \notin$ $C_{i}$. Therefore, $v \in T_{i} \backslash C_{i}$. It follows that there exist $s_{1}, \ldots, s_{p} \in \widetilde{S}$ such that $T_{i} \backslash C_{i}=\bigcup_{k=1}^{p} T_{s_{k}}$, and where $\left|\operatorname{supp}\left(C_{s_{j}}\right)\right|>\left|\operatorname{supp}\left(C_{i}\right)\right|, j \in\langle p\rangle$. By (2.10) and the inductive assumption we obtain

$$
X_{T_{i} \backslash C_{i}} \leq \max _{k \in\langle p\rangle}\left\{X_{T_{s_{k}}}\right\} \leq \max _{(j, k) \in\left(S \times S^{c}\right) \cap E}\left\{\min _{\substack{T \subseteq\langle n) \\(j, k) \in T \times T^{c}}}\left\{X_{T}\right\}\right\}
$$

and by (2.21) we obtain (2.15).

Observe that $\widetilde{S} \subseteq \widehat{S},=\bigcup_{i \in S} T_{i} \subseteq S$. Substitution of the sets $S, S^{c}$, and $\widehat{S}$ for the sets $S, T$, and $S^{\prime}$ in (2.9) respectively yields $X_{S} \leq X_{\hat{S}}$. By (2.10) we obtain $X_{S} \leq \max _{i \in \widetilde{S}^{S}}\left\{X_{T_{i}}\right\}$, and by (2.15) we have

$$
X_{S} \leq \max _{(j, k) \in\left(S \times S^{c}\right) \cap E}\left\{\min _{\substack{T \subseteq\langle n) \\(j, k) \in T \times T^{c}}}\left\{X_{T}\right\}\right\}
$$

This proves our claim. It now follows from (2.13) and (2.22) that $\max _{(i, j) \in S \times S^{\mathrm{c}} \cap E}\left\{a_{i j}\right\}=X_{S}, S \subseteq\langle n\rangle$.

Let $S \subseteq\langle n\rangle$. If we choose $T$ in $(2.9)$ to be $S^{c}$, then we get

$$
X_{S} \leq X_{S^{\prime}}, \quad \widetilde{S} \subseteq S^{\prime} \subseteq S .
$$

Another weaker form of $(2.9)$ is

$$
X_{S} \leq \max \left\{X_{S \cup T}, X_{\widetilde{S}_{T}}\right\}, \quad S, T \subseteq\langle n\rangle, \quad S \cap T=\emptyset .
$$

It is natural to ask whether the condition (2.9) in Theorem 2.6 can be replaced by (2.23) and/or (2.24). The answer to this question is negative, as demonstrated by the following example.

EXAMPLE 2.25. Let $n=4$, and let $D$ be the digraph with four vertices and all possible arcs except for $(2,4)$ and $(3,4)$. For a subset $S$ of $\langle 4\rangle$ define

$$
X_{S}=\left\{\begin{array}{rr}
-\infty, & S=\emptyset,\langle 4\rangle \\
1, & S=\{1,3\} \\
2, & \text { otherwise }
\end{array}\right.
$$


The only $S \subseteq\langle 4\rangle$ for which $S \neq \widetilde{S}$ is $S=\{1,2,3\}$, where $\widetilde{S}=\{1\}$. Since $X_{\{1,2,3\}}=X_{\{1\}}=2,(2.23)$ is satisfied. Now, let $S, T \subseteq\langle 4\rangle, S \cap T=\emptyset$. Observe that we have $S \neq \widetilde{S}_{T}$ if and only if $T=\{4\}$ and $\{2,3\} \cap S \neq \emptyset$. In these cases we have

$$
\begin{aligned}
& S=\{2\},\{3\},\{1,2\},\{2,3\},\{1,3\} \quad \Rightarrow \quad X_{S}=2=X_{S \cup T}, \\
& S=\{1,2,3\} \Rightarrow \widetilde{S}_{T}=\{1\} \quad \Rightarrow \quad X_{S}=2=X_{\widetilde{S}_{T}}
\end{aligned}
$$

and hence (2.24) is satisfied. Finally, it is immediate to check that (2.10) and (2.11) are satisfied. Nevertheless, if we choose $S=\{1,2,3\}, T=$ $\{4\}$, and $S^{\prime}=\{1,3\}$, then $\widetilde{S}_{T}=\{1\} \subseteq S^{\prime} \subseteq S$ and $X_{S}=2>1=$ $\max \left\{X_{S \cup T}, X_{\widetilde{S}_{T}}\right\}$. Therefore, (2.9) is not satisfied, and by Theorem 2.6 there exists no $n \times n$ matrix $A$, with $D(A) \subseteq D$, satisfied (2.7).

With the convention that the minimum of the elements of the empty subset of the reals will be considered as $\infty$, we obtain the following corollary of Theorem 2.6.

THeorem 2.26. Let $\left\{X_{S}: S \subseteq\langle n\rangle\right\}$ be a set of numbers, and let $D=(\langle n\rangle, E)$ be a digraph. The following are equivalent:

(i) There exists an $n \times n$ matrix $A$, with $D(A) \subseteq D$, such that

$$
\min _{(i, j) \in S \times S^{c} \cap E}\left\{a_{i j}\right\}=X_{S}, \quad S \subseteq\langle n\rangle .
$$

(ii) We have

$$
X_{\emptyset}=X_{\langle n\rangle}=\infty
$$

$$
\begin{aligned}
X_{S} & \geq \min \left\{X_{S \cup T}, X_{S}\right\}, & & S, T \subseteq\langle n\rangle, S \cap T=\emptyset, \widetilde{S}_{T} \subseteq S^{\prime} \subseteq S, \\
X_{S \cup T} & \geq \min \left\{X_{S}, X_{T}\right\}, & & S, T \subseteq\langle n\rangle, \\
X_{S \cap T} & \geq \min \left\{X_{S}, X_{T}\right\}, & & S, T \subseteq\langle n\rangle .
\end{aligned}
$$

Proof. A matrix $A$ satisfies $\min _{(i, j) \in S \times S^{c} \cap E}\left\{a_{i j}\right\}=X_{S}, S \subseteq\langle n\rangle$, if and only if the matrix $B=-A$ satisfies $\max _{(i, j) \in S \times S^{c} \cap E}\left\{b_{i j}\right\}=-X_{S}$ whenever $S \subseteq\langle n\rangle$. Our claim now follows from Theorem 2.6.

Theorems 2.6 and 2.26 provide a necessary and sufficient condition for the existence of a matrix $A$, with $D(A) \subseteq D$, with prescribed off-diagonal block element maxima or minima. The case $D(A)=D$ is covered by the following corollaries. 
COROLLARY 2.27. Let $\left\{X_{S}: S \subseteq\langle n\rangle\right\}$ be a set of real numbers, and let $D=(\langle n\rangle, E)$ be a digraph. The following are equivalent:

(i) There exists an $n \times n$ matrix $A$, with $D(A)=D$, such that

$$
\max _{(i, j) \in S \times S^{c} \cap E}\left\{a_{i j}\right\}=X_{S}, \quad S \subseteq\langle n\rangle .
$$

(ii) We have (2.8), (2.9), (2.10), (2.11), and

$$
X_{S} \neq 0 \quad \text { for every relevant subset } S \text { of }\langle n\rangle \text {. }
$$

Proof. (i) $\Rightarrow$ (ii): (2.8), (2.9), (2.10), and (2.11) follow from (i) by Theorem 2.6. Since $D(A)=D, \max _{(i, j) \in S \times S^{c} \cap E}\left\{a_{i j}\right\} \neq 0$ whenever $S$ is relevant. (2.28) now follows from (i).

(ii) $\Rightarrow$ (i): In view of (2.28), the matrix defined by (2.12), constructed in the proof of the corresponding implication in Theorem 2.5 , satisfies $D(A)=$ $D$.

Similarly, we obtain

COROLlaRY 2.29. Let $\left\{X_{S}: S \subseteq\langle n\rangle\right\}$ be a set of real numbers, and let $D=(\langle n\rangle, E)$ be a digraph. The following are equivalent:

(i) There exists an $n \times n$ matrix $A$, with $D(A)=D$, such that

$$
\min _{(i, j) \in S \times S^{\circ} \cap E}\left\{a_{i j}\right\}=X_{S}, \quad S \subseteq\langle n\rangle .
$$

(ii) We have (2.8), (2.9), (2.10), (2.11), and (2.28).

The following two corollaries are the "graph-free" versions of our results.

Corollary 2.30. Let $\left\{X_{S}: S \subseteq\langle n\rangle, S \neq \emptyset,\langle n\rangle\right\}$ be a set of numbers. The following are equivalent:

(i) There exists an $n \times n$ matrix $A$ such that

$$
\max _{(i, j) \in S \times S^{c}}\left\{a_{i j}\right\}=X_{S}, \quad S \subseteq\langle n\rangle, \quad S \neq \emptyset,\langle n\rangle .
$$

(ii) For every nonempty proper subsets $S$ and $T$ of $\langle n\rangle$ we have $X_{S \cup T}$ $\leq \max \left\{X_{S}, X_{T}\right\}$ whenever $S \cup T \neq\langle n\rangle$, and $X_{S \cap T} \leq \max \left\{X_{S}, X_{T}\right\}$ whenever $S \cap T \neq \emptyset$. 
COROLlaRY 2.31. Let $\left\{X_{S}: S \subseteq\langle n\rangle, S \neq \emptyset,\langle n\rangle\right\}$ be a set of numbers. The following are equivalent:

(i) There exists an $n \times n$ matrix $A$ such that

$$
\min _{(i, j) \in S \times S^{c}}\left\{a_{i j}\right\}=X_{S}, \quad S \subseteq\langle n\rangle, \quad S \neq \emptyset,\langle n\rangle .
$$

(ii) For every nonempty proper subsets $S$ and $T$ of $\langle n\rangle$ we have $X_{S \cup T}$ $\geq \min \left\{X_{S}, X_{T}\right\}$ whenever $S \cup T \neq\langle n\rangle$, and $X_{S \cap T} \geq \min \left\{X_{S}, X_{T}\right\}$ whenever $S \cap T \neq \emptyset$.

REMARK 2.32. The graph-free case could be proven directly rather easily. The implication (i) $\Rightarrow$ (ii) in Corollary 2.30 follows as before. As to the implication (ii) $\Rightarrow$ (i), we define $a_{i j}$ as in (2.12) and derive (2.13). Now suppose that for some nonempty proper subset $S$ of $\langle n\rangle$ we have

$$
\max _{(i, j) \in S \times S^{c}}\left\{a_{i j}\right\}<X_{S} .
$$

It follows by (2.12) and (2.13) that

$$
\max _{(i, j) \in S \times S^{c}}\left\{T_{i j}\right\}<X_{S}
$$

Note that

$$
\bigcup_{i \in S}\left(\bigcap_{j \in S^{c}} T_{i j}\right)=S .
$$

Therefore, it follows from (2.33) and (2.34) by (2.10) and (2.11) that

$$
X_{S} \leq \max _{(i, j) \in S \times S^{c}}\left\{T_{i j}\right\}<X_{S},
$$

which is a contradiction.

However, in general we cannot employ such a simple proof, as we do not have an analogue of (2.34).

REMARK 2.35. Observe that in the case of nonnegative $X_{S}$ 's, the matrix A defined by (2.12) is nonnegative. Therefore, if the numbers $\left\{X_{S}: S \subseteq\langle n\rangle\right\}$ are given to be nonnegative, then our results provide necessary and sufficient conditions for the existence of a nonnegative matrix $A$ satisfying the required conditions. 


\section{THE UNIQUENESS PROBLEM}

The existence problems discussed in the previous section do not necessarily yield a unique solution, as demonstrated by the following example.

EXAMPLE 3.1. Let $n=4$, let $D$ be the complete (loopless) digraph with four vertices, and let

$$
\begin{gathered}
X_{\emptyset}=X_{\langle 4\}}=-\infty, \\
X_{\{1\}}=3, \quad X_{\{2\}}=7, \quad X_{\{3\}}=9, \quad X_{\{4\}}=12, \\
X_{\{1,2\}}=5, X_{\{1,3\}}=9, X_{\{2,3\}}=8, X_{\{1,4\}}=12, X_{\{2,4\}}=12, X_{\{3,4\}}=11, \\
X_{\{1,2,3\}}=6, \quad X_{\{1,2,4\}}=12, X_{\{1,3,4\}}=11, X_{\{2,3,4\}}=10 .
\end{gathered}
$$

The numbers $\left\{X_{S}: S \subseteq\langle 4\rangle\right\}$ satisfy (2.8), (2.9), (2.10), and (2.11). The matrix

$$
A=\left(\begin{array}{cccc}
0 & 3 & 3 & 3 \\
7 & 0 & 5 & 5 \\
8 & 9 & 0 & 6 \\
10 & 11 & 12 & 0
\end{array}\right)
$$

defined by (2.12), satisfies (2.7). However, (2.7) is satisfied also by

$$
A=\left(\begin{array}{cccc}
0 & 1 & 2 & 3 \\
7 & 0 & 4 & 5 \\
8 & 9 & 0 & 6 \\
10 & 11 & 12 & 0
\end{array}\right)
$$

In this section we study the uniqueness of the solutions for the problems discussed in the previous section. For a digraph $D$ we denote by $E(D)$ and $V(D)$ the arc set and the vertex set of $D$ respectively. Our problem can be stated as follows:

Given an $n \times n$ matrix $A$. Does there exist another $n \times n$ matrix $B$, with $D(B) \subseteq D(A)$, such that

$$
\max _{(i, j) \in S \times S^{c} \cap E(D(A))}\left\{b_{i j}\right\}=\max _{(i, j) \in S \times S^{c} \cap E(D(A))}\left\{a_{i j}\right\}
$$

for every relevant subset $S$ of $V(D(A))$ ?

We shall define the ROWB algorithm for removal of certain arcs in a digraph, and we shall use it to characterize those graphs $D$ such that for every matrix $A$ with $D(A)=D$, there exists no other matrix $B$ with $D(B) \subseteq D$ satisfying (3.2), as well as those graphs $D$ such that for some 
matrix $A$ with $D(A)=D$, there exists no other matrix $B$ with $D(B) \subseteq D$ satisfying (3.2).

DEFINITION 3.3. An arc $(i, j)$ in a digraph $D=(V, E)$ is said to be a one-way bridge if there exists a set $S$ of vertices in $D$ such that $(i, j)$ is the only arc from $S$ to $S^{c}$.

The following easy proposition can be regarded as an alternative definition for one-way bridges.

Proposition 3.4. An arc $(i, j)$ in a digraph $D$ is a one-way bridge if and only if the digraph obtained by removing $(i, j)$ from $D$ has no path from $i$ to $j$.

Proof. If $(i, j)$ is a one-way bridge, then by Definition 3.3 there exists a set $S$ of vertices in $D$ such that $(i, j)$ is the only arc from $S$ to $S^{c}$. It follows that the digraph obtained by removing $(i, j)$ from $D$ has no path from $i$ to $j$. Conversely, let $D^{\prime}$ be the digraph obtained by removing $(i, j)$, and let $S$ be the set of vertices $k$ such that there exists a path from $i$ to $k$ in $D^{\prime}\left(S\right.$ includes $i$ ). If $D^{\prime}$ has no path from $i$ to $j$, then $j$ belongs to $S^{c}$. Clearly, there is no arc in $D^{\prime}$ from $S$ to $S^{c}$. Therefore, $(i, j)$ is the only arc from $S$ to $S^{c}$.

DEFINITION 3.5. The ROWB algorithm (removal of one-way bridges):

Initialization: We let $D_{1}$ be the digraph $D$.

Step $i, i=1, \ldots$ : We remove all the one-way bridges from $D_{i}$, and we denote the resulting digraph by $D_{i+1}$.

The ROWB algorithm terminates after $k-1$ steps if $D_{k}$ has no one-way bridges. We then denote $D_{k}$ by $\breve{D}$. The ROWB index of $D$ is defined to be equal to $k-1$ if $\breve{D}$ has no arcs, and is $\infty$ otherwise. Clearly, $\breve{D}$ has no one-way bridges.

\section{EXAMPLE 3.6.}

(i) It is easy to verify that any digraph consisting of one cycle has ROWB index 1. Also, the ROWB index of any digraph $D$, such that the (undirected) graph obtained by removing the directions from the arcs of $D$ is a tree, is equal to 1 . Clearly, any digraph consisting of disjoint digraphs of $R O W B$ index 1 has $R O W B$ index 1 . 
(ii) The one-way bridges in the graph

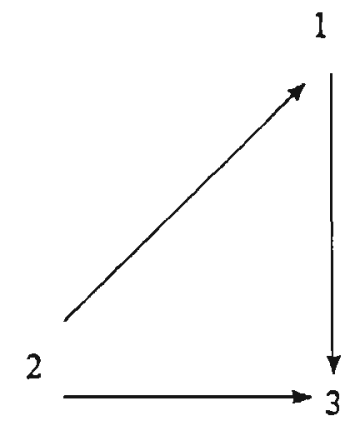

are $(1,3)$ and $(2,1)$. Therefore, the only arc in $D_{2}$ is $(2,3)$, and, being a one-way bridge in $D_{2}$, it gets removed in the second step. Thus, the $R O W B$ index of $D$ is 2 .

(iii) It is easy to verify that for any complete digraph $D$ with more than two vertices we have $\check{D}=D$, and so $D$ has $R O W B$ index $\infty$. Also, every digraph with multiple arcs has $R O W B$ index $\infty$.

We can now characterize those patterns that yield a unique solution for every set of given maxima.

THEOREM 3.7. Let $D=(\langle n\rangle, E)$ be a digraph. The following are equivalent:

(i) For every $n \times n$ matrix $A$ with $D(A) \subseteq D$, there exists no other $n \times n$ matrix $B$ with $D(B) \subseteq D$ satisfying (3.2).

(ii) For every $n \times n$ matrix $A$ with $D(A)=D$, there exists no other $n \times n$ matrix $B$ with $D(B) \subseteq D$ satisfying (3.2)

(iii) The ROWB index of $D$ is less than or equal to 1.

Proof. (i) $\Rightarrow$ (ii) is trivial.

(ii) $\Rightarrow$ (iii): Assume that (iii) does not hold, that is, some $\operatorname{arc}(k, l)$ in $D(A)$ is not a one-way bridge in $D(A)$. Define a matrix $A_{\varepsilon}$, of the same size as $A$, by

$$
\left(A_{\epsilon}\right)_{i j}= \begin{cases}1, & (i, j) \in E(D), \quad(i, j) \neq(k, l) \\ \epsilon, & (i, j)=(k, l), \\ 0 & \text { otherwise }\end{cases}
$$


where $\epsilon \leq 1$. Let $S$ be a nonempty proper subset of vertices of $D$. If there are no $\operatorname{arcs}$ from $S$ to $S^{c}$, then

$$
\max _{(i, j) \in S \times S^{c} \cap E(D(A))}\left\{\left(A_{\epsilon}\right)_{i j}\right\}=0 .
$$

If there are arcs from $S$ to $S^{c}$, then, since $(i, j)$ cannot be the only arc from $S$ to $S^{c}$ and since $\epsilon \leq 1$, it follows from (3.8) that

$$
\max _{(i, j) \in S \times S^{c} \cap E(D(A))}\left\{\left(A_{\epsilon}\right)_{i j}\right\}=1 .
$$

Thus, all $A_{\epsilon}$ share the same set

$$
\left\{\max _{(i, j) \in S \times S^{c} \cap E(D(A))}\left\{\left(A_{\epsilon}\right)_{i j}\right\}: S \subseteq\langle n\rangle\right\},
$$

in contradiction to (ii).

(iii) $\Rightarrow$ (i): Let $A$ and $B$ be $n \times n$ matrices, with $D(A) \subseteq D$ and $D(B) \subseteq D$, satisfying (3.2). If the ROWB index of $D$ is less than or equal to 1 , then every arc $(k, l)$ in $D$ is a one-way bridge, that is, there exists a set $S$ of vertices in $D$ such that $(k, l)$ is the only arc from $S$ to $S^{c}$. Therefore, from (3.2) we obtain

$$
\max _{(i, j) \in S \times S^{c} \cap E(D(B))}\left\{b_{i j}\right\}=b_{k l}=\max _{(i, j) \in S \times S^{c} \cap E(D(A))}\left\{a_{i j}\right\}=a_{k l},
$$

and so $A=B$.

The characterizations of the patterns that yield a unique solution of some set of given maxima is as follows.

THEOREM 3.9. Let $D=(\langle n\rangle, E)$ be a digraph. The following are equivalent:

(i) For some $n \times n$ matrix $A$ with $D(A)=D$, there exists no other $n \times n$ matrix $B$ with $D(B) \subseteq D$ satisfying (3.2)

(ii) The ROWB index of $D$ is finite.

Proof. (i) $\Rightarrow$ (ii): Assume that the $R O W B$ index of $D$ is $\infty$, and let $A$ be any matrix with. $D(A)=D$. As $\check{D}$ has arcs, let $(k, l)$ be an $\operatorname{arc}$ in $\check{D}$ such that $a_{k l}$ is minimal. For $\epsilon>0$ we define a matrix $A_{\epsilon}$, of the same size as $A$, by

$$
\left(A_{\epsilon}\right)_{i j}= \begin{cases}a_{i j}, & (i, j) \neq(k, l), \\ a_{k l}-\epsilon, & (i, j)=(k, l) .\end{cases}
$$


Let $S$ be a set of vertices such that $(k, l) \in S \times S^{c}$. Since $\breve{D}$ has no one-way bridges, it follows that there exists at least one more arc in $\check{D}$ from $S$ to $S^{c}$. It now follows from (3.10), by the minimality of $a_{k l}$, that

$$
\begin{aligned}
& \max _{(i, j) \in S \times S^{c} \cap E(D(B))}\left\{\left(A_{\epsilon}\right)_{i j}\right\} \\
& =\max _{(i, j) \in S \times S^{c} \cap E(D(A))}\left\{a_{i j}\right\} \quad \text { for every } S \subseteq\langle n\rangle,
\end{aligned}
$$

in contradiction to (i).

(ii) $\Rightarrow$ (i): We define a matrix $A$, with $D(A)=D$, as follows. For each $(k, l) \in E(D)$ we let $a_{k l}=m$, where $m$ is the number of the step of the ROWB algorithm in which $(k, l)$ gets removed from $D$. Now, let $B$ an $n \times n$ matrix, with $D(B) \subseteq D$, satisfying (3.2), and let $(k, l)$ be an arc in $D$. We prove that $a_{k l}=b_{k l}$ by induction on the number $m$ of the step of the ROWB algorithm in which $(k, l)$ gets removed from $D$. If $m=1$, then there exists a set $S$ of vertices in $D$ such that $(k, l)$ is the only arc from $S$ to $S^{c}$, and from (3.2) we obtain

$$
\max _{(i, j) \in S \times S^{c} \cap E(D(B))}\left\{b_{i j}\right\}=b_{k l}=\max _{(i, j) \in S \times S^{\complement} \cap E(D(A))}\left\{a_{i j}\right\}=a_{k l} .
$$

Assume that our claim holds for $m<p$ where $p>1$, and let $m=p$. Since $(k, l)$ gets removed from $D$ in the pth step of the ROWB algorithm, it follows that there exists a set $S$ of vertices in $D$ such that $(k, l)$ is an arc from $S$ to $S^{c}$, and every other arc from $S$ to $S^{c}$ gets removed in an earlier step. By the inductive assumption we have

$$
b_{i j}=a_{i j}<p \quad \text { for every } \quad(i, j) \in S \times S^{c}, \quad(i, j) \neq(k, l) .
$$

Since by (3.2) we have

$$
\max _{(i, j) \in S \times S^{c} \cap E(D(B))}\left\{b_{i j}\right\}=\max _{(i, j) \in S \times S^{C} \cap E(D(A))}\left\{a_{i j}\right\}=p,
$$

it now follows from (3.11) that we necessarily have $a_{k l}=b_{k l}$.

We conclude this section with a description of all possible solutions to the existence problem of matrices with off-diagonal block element maximum.

Definition 3.12. Let $\mathcal{X}=\left\{X_{S}: S \subseteq\langle n\rangle, S \neq \emptyset,\langle n\rangle\right\}$ be a set of numbers, and let $D=(\langle n\rangle, E)$ be a digraph. Let $A$ be the matrix defined by (2.12). A set $\mathcal{P}$ of arcs in $D$ is said to be $\mathcal{X}$-complete if for every relevant subset $S$ of $\langle n\rangle$ there exists $(i, j) \in S \times S^{c} \cap P$ such that $a_{i j}=X_{S}$. 
THEOREM 3.13. Let $\mathcal{X}=\left\{X_{S}: S \subseteq\langle n\rangle, S \neq \emptyset,\langle n\rangle\right\}$ be a set of numbers, and let $D=(\langle n\rangle, E)$ be a digraph. Let $A$ be the matrix defined by (2.12). The following are equivalent:

(i) An $n \times n$ matrix $B$ satisfies

$$
\max _{(i, j) \in S \times S^{c} \cap E}\left\{b_{i j}\right\}=X_{S} \quad \text { for every relevant subset } S \text { of }\langle n\rangle .
$$

(ii) There exists an $\mathcal{X}$-complete set $\mathcal{P}$ of arcs in $D$ such that $b_{i j}=a_{i j}$ whenever $(i, j) \in \mathcal{P}$ and $b_{i j} \leq a_{i j}$ whenever $(i, j) \in E \backslash \mathcal{P}$.

Proof. (i) $\Rightarrow$ (ii): Let $(i, j) \in E$, and let $S$ be a subset of $\langle n\rangle$ such that

$$
(i, j) \in S \times S^{c} \quad \text { and } \quad X_{S}=\min _{\substack{T \subseteq(n) \\(i, j) \in\left(\bar{T} \times T^{c}\right) \cap E}}\left\{X_{T}\right\} .
$$

We have $a_{i j}=X_{S}=\max _{(k, l) \in S \times S^{c} \cap E}\left\{b_{k l}\right\} \geq b_{i j}$. Also, for every relevant subset $S$ of $\langle n\rangle$ there exists $\left(i_{S}, j_{S}\right) \in S \times S^{c} \cap E$ such that $b_{i_{S} j_{S}}=X_{S}$, and since $X_{S} \geq a_{i_{S} j_{S}} \geq b_{i_{S} j_{S}}=X_{S}$, we have $a_{i_{S j_{S}}}=b_{i_{S} j_{S}}$. Therefore, $P=\left\{\left(i_{S}, j_{S}\right): S\right.$ is a relevant subset of $\left.\langle n\rangle\right\}$ is an $x$-complete set.

(ii) $\Rightarrow$ (i): Let $S$ be a relevant subset of $\langle n\rangle$. It follows from (ii) that

$$
\max _{(i, j) \in S \times S^{c} \cap E(D(A))}\left\{b_{i j}\right\} \leq \max _{(i, j) \in S \times S^{c} \cap E(D(A))}\left\{a_{i j}\right\}=X_{S} .
$$

Furthermore, since $\mathcal{P}$ is an $x$-complete set, there exists $(i, j) \in S \times S^{c} \cap \mathcal{P}$ such that $a_{i j}=X_{S}$. By (ii) we have $b_{i j}=a_{i j}=X_{S}$, and so $\max _{(i, j) \in S \times S^{\mathrm{c}} \cap E}\left\{b_{i j}\right\}=$ $X_{S}$.

\section{DIGRAPHS WITH ROWB INDEX 1}

Motivated by Theorem 3.7, we include here a short discussion of digraphs with $R O W B$ index 1 . Note that a digraph has $R O W B$ index 1 if and only if every arc in the graph is a one-way bridge.

Definition 4.1. Let $D$ be a digraph.

(i) A digraph $D$ is said to be strongly connected if for every two vertices $i$ and $j$ in $D$ there is a path from $i$ to $j$ in $D$.

(ii) A subdigraph $S$ of $D$ is said to be a strong component if $S$ is a maximal strongly connected subdigraph of $D$. 
(iii) A digraph $D$ is said to be minimal strongly connected if $D$ is strongly connected and no digraph obtained by removing an arc from $D$ is strongly connected.

DEFINITION 4.2. The condensation $D^{*}$ of a digraph $D$ is a digraph whose vertices are the strong components $S_{1}, \ldots, S_{m}$ of $D$, and where $\left(S_{i}, S_{j}\right)$ is an arc in $D^{*}$ with multiplicity $k$ if there are exactly $k$ arcs from vertices in $S_{i}$ to vertices in $S_{j}$.

THEOREM 4.3. Let $D$ be a digraph. The following are equivalent:

(i) $D$ has ROWB indeed.1.

(ii) The strong components of $D$, as well as the condensation $D^{*}$, have ROWB index 1.

Proof. (i) $\Rightarrow$ (ii): Clearly, if $D$ has $R O W B$ index 1 , then every subdigraph of $D$ has $R O W B$ index 1 , and, in particular, the strong components of $D$ have $R O W B$ index 1 . Now, let $\left(S_{i}, S_{j}\right)$ be an arc in $D^{*}$. By Definition 4.2 , there exist a vertex $u$ in $S_{i}$ and a vertex $v$ in $S_{j}$ such that $(u, v)$ is an arc in $D$. Since $D$ has $R O W B$ index $1,(u, v)$ is a one-way bridge, and by Proposition 3.4, the digraph obtained by removing $(u, v)$ from $D$ has no path from $u$ to $v$. It follows that $\left(S_{i}, S_{j}\right)$ is a simple arc in $D^{*}$ and that the digraph obtained by removing $\left(S_{i}, S_{j}\right)$ from $D^{*}$ has no path from $S_{i}$ to $S_{j}$. By Proposition 3.4, $D^{*}$ has $R O W B$ index 1 .

(ii) $\Rightarrow$ (i): Let $(u, v)$ be an $\operatorname{arc}$ in $D$. If $u$ and $v$ belong to the same strong component of $D$, then, since the strong components of $D$ have $R O W B$ index 1 , it follows that the digraph obtained by removing $(u, v)$ from $D$ has no path from $u$ to $v$, and by Proposition 3.4, $(u, v)$ is a one-way bridge in $D$. Assume now that $u$ and $v$ belong to two different strong components $S_{i}$ and $S_{j}$ of $D$ respectively. Since $D^{*}$ has $R O W B$ index $1,\left(S_{i}, S_{j}\right)$ is a one-way bridge in $D^{*}$, and it follows that $(u, v)$ is the only arc in $D$ from a vertex in $S_{i}$ to a vertex in $S_{j}$, and that the digraph obtained by removing $\left(S_{i}, S_{j}\right)$ from $D^{*}$ has no path from $S_{i}$ to $S_{j}$. It follows that the digraph obtained by removing $(u, v)$ from $D$ has no path from $u$ to $v$, and by Proposition $3.4,(u, v)$ is a one-way bridge in $D$.

Theorem 4.3 reduces the problem of characterizing digraphs of $R O W B$ index 1 to the problem of characterizing strongly connected digraphs with $R O W B$ index 1 (the strong components of $D$ ) as well as acyclic digraphs with $R O W B$ index 1 (the condensation $D^{*}$ ). We conclude the paper with a proposition that handles the strongly connected case. 
Proposition 4.4. A strongly connected digraph $D$ has $R O W B$ index 1 if and only if $D$ is minimal strongly connected.

Proof. Let $D$ be a minimal strongly connected digraph, and let $(i, j)$ be an arc in $D$. Observe that, since $D$ is strongly connected, if the digraph $D^{\prime}$ obtained by removing $(i, j)$ from $D$ has a path from $i$ to $j$, then $D^{\prime}$ too is strongly connected. Thus, since $D$ is minimal strongly connected, it follows that $D^{\prime}$ has no path from $i$ to $j$, and by Proposition $3.4,(i, j)$ is a one-way bridge in $D$. Hence, $D$ has ROWB index 1 . Conversely, assume that $D$ has $R O W B$ index 1 , and let $(i, j)$ be an $\operatorname{arc}$ in $D$. By Proposition 3.4, the digraph obtained by removing $(i, j)$ from $D$ has no path from $i$ to $j$, and so it is not strongly connected. Therefore, $D$ is minimal strongly connected.

\section{REFERENCES}

1 R. A. Brualdi, Convex sets of non-negative matrices, Canad. J. Math. 20:144-157 (1968).

2 J. A. Dias Da Silva, D. Hershkowitz, and H. Schneider, Existence of matrices with prescribed off-diagonal block element sums, to appear.

$3 \mathrm{M}$. V. Menon and H. Schneider, The spectrum of a nonlinear operator associated with a matrix, Canad. J. Math. 20:225-232 (1967).

4 U. G. Rothblum and H. Schneider, Scalings of matrices which have prespecified row and column sums via optimization, Linear Algebra Appl. 114/115:737-767 (1989).

5 U. G. Rothblum, H. Schneider, and M. H. Schneider, Scaling matrices to prescribed row and column maxima, SIAMJ. Matrix Anal. Appl., to appear.

6 H. Schneider and M. H. Schneider, Max-balancing weighted directed graphs and matrix scaling, Math. Oper. Res. 16:208-222 (1991).

7 R. Sinkhorn, A relationship between arbitrary positive matrices and stochastic matrices, Ann. Math. Statist. 35:876-879 (1964). 